Classification

Physics Abstracts

$08.00-07.20-18.10$

\title{
STABILISATION PAR EFFET PÖCKELS DE LA PUISSANCE D'UN FAISCEAU LASER
}

\author{
J. C. LOPEZ, N. DAUDE et Ch. GRECO \\ Laboratoire de Physique de l'Etat Cristallin \\ 2. Université des Sciences et Techniques du Languedoc \\ 34-Montpellier, France \\ (Reçu le 18 février 1972, révisé le 7 juin 1973)
}

\begin{abstract}
Résumé. - Le montage décrit permet de stabiliser à $\pm 1 \%$ la puissance transportée par un faisceau laser. Les fluctuations de l'intensité lumineuse sont compensées par des variations asservies de la transparence d'un cristal de KDP placé entre deux polariseurs parallèles.

Abstract. - A device allowing a relative stability of about $1 \%$ of the laser beam power is described. The fluctuations of light intensity are servo-compensated by varying the transparency of a KDP crystal between parallel polarizers.
\end{abstract}

1. Introduction. - La stabilité de la source lumineuse est un critère primordial dans la plupart des études spectrométriques : elle est indispensable pour les mesures d'intensité des raies spectrales. A l'exception de certains lasers très coûteux, qui comportent des dispositifs de stabilisation, les tubes de grande puissance - lasers à argon ionisé en particulier présentent des instabilités qui peuvent atteindre $\pm 20 \%$. Le montage décrit ci-dessous stabilise le flux transporté par le faisceau sans modifier l'intensité lumineuse délivrée par le laser. Il ne nécessite aucune transformation de tube ou de l'alimentation. L'asservissement agit sur une source de tension de faible puissance et possède une très faible inertie.

2. Principe de fonctionnement. -2.1 Dispositif optiQue (CEllule de PöCKels). - On interpose sur le trajet du faisceau laser une lame de KDP taillée perpendiculairement à l'axe $O Z$ et placée entre les armatures d'un condensateur plan. Les plaques conductrices sont percées d'un trou permettant le passage du faisceau et suffisamment étroit pour ne pas perturber le champ électrique uniforme. Le faisceau lumineux traverse ensuite un analyseur dont le plan de polarisation est parallèle à celui du faisceau laser.

2.2 RéGulation électronique. - Le dispositif de régulation agissant sur le cristal de KDP est commandé par un élément photosensible (photorésistance LDRO5) qui reçoit une partie du flux lumineux à corriger. Après amplification, le signal d'erreur modifie la tension de grille d'un tube électronique qui fixe la tension appliquée au cristal de KDP.

Le coefficient de stabilisation $\chi$ (rapport des fluctuations relatives à la sortie et à l'entrée) est égal à

$$
\chi=1-\lambda \Delta V \cdot\left(\frac{\Delta \Phi_{\mathrm{E}}}{\Phi_{\mathrm{E}}}\right)^{-1}
$$

$\Phi_{\mathrm{E}}$ : flux entrant $; \Phi_{\mathrm{S}}$ : flux sortant $; \lambda=2 \alpha \operatorname{tg} \alpha . V$ où

$$
\alpha=\frac{\pi n_{0}^{3}}{\lambda_{0}} r_{63}
$$

et $V:$ différence de potentiel aux bornes du cristal; $\Delta V$ : variable de tension à la sortie de l'amplificateur de gain $G$, est donné par

$$
\Delta V=G \Delta V_{\mathrm{E}}
$$

en appelant $\Delta V_{E}$ la variation de tension à l'entrée de l'amplificateur.

Cette variation de tension est provoquée par la variation de résistance de la photorésistance montée dans une branche de potentiomètre. L'étude expérimentale des variations de résistance en fonction du flux reçu montre que $R=k / \Phi$ où $k$ est une constante caractéristique de la photorésistance.

Il en résulte :

$$
\frac{\Delta R}{R}=-\frac{\Delta \Phi_{\mathrm{S}}}{\Phi_{\mathrm{S}}}
$$

On peut calculer $\Delta V_{\mathrm{E}}$ en fonction des caractéristiques du circuit ; en effet :

$$
V_{\mathrm{E}}=\frac{V_{\mathrm{cc} \rho}}{R+\rho} \simeq \frac{V_{\mathrm{cc} \rho}}{R} \text { si } \rho \ll R
$$

$V_{\mathrm{cc}}$ : tension continue appliquée au potentiomètre, $R$ : valeur de la résistance de la photorésistance, $\rho$ : valeur de la $2^{\mathrm{e}}$ résistance du potentiomètre d'entrée.

Soit $V_{\mathrm{E}_{0}}=V_{\mathrm{cc}} \rho / R_{0}$ la valeur initiale de la tension d'entrée. Il vient d'après (3) et (4) :

$$
\Delta V_{\mathrm{E}}=-\frac{V_{\mathrm{cc} \rho}}{R_{0}} \cdot \frac{\Delta R}{R_{0}}=V_{\mathrm{E}_{0}} \cdot \frac{\Delta \Phi_{\mathrm{S}}}{\Phi_{\mathrm{S}}} .
$$


L'expression (5) devient, en tenant compte de (2) et (5)

$$
\chi=1-\lambda G V_{\mathrm{E}_{0}}\left(\frac{\Delta \Phi_{\mathrm{S}}}{\Phi_{\mathrm{S}}}\right)\left(\frac{\Delta \Phi_{\mathrm{E}}}{\Phi_{\mathrm{E}}}\right)^{-1}
$$

d'où :

$$
\chi=\frac{1}{\lambda G V_{\mathrm{E}_{0}}} \text { si } \lambda G V_{\mathrm{E}_{0}} \gg 1 .
$$

Les variations relatives de flux sont divisées par $\lambda G V_{\mathrm{E}_{0}}$ qui peut être rendu très grand.

3. Résultats et discussion. - 3.1 CHOIX DES CARACTÉRISTIQUES. - Pour le laser LGA 1098 CSF en notre possession, l'enregistrement de l'intensité lumineuse montre que l'écart quadratique moyen est de l'ordre de $15 \%$ en valeur relative en l'absence de correction (Fig. 1a).

Le procédé expérimental est le suivant: après 20 min environ, la très forte dérive due à la mise en température disparaît. La résistance $\rho$ est alors réglée pour que la tension appliquée au cristal de KDP soit voisine de $2000 \mathrm{~V}$. Le montage électronique fournissant une tension utile de $3500 \mathrm{~V}$ au maximum, le dispositif peut corriger des fluctuations d'amplitude maximale $15 \%$.

3.2 EXPÉRIENCES RÉALISÉES ET DISCUSSION. - Les différents enregistrements effectués sont rassemblés dans la figure 1. La courbe supérieure de chaque enregistrement montre les variations de la puissance émise par le laser. Le flux sortant de la cellule de Pöckels est enregistré simultanément sur la courbe inférieure.

La figure $1 a$ montre les variations des deux signaux sans correction: c'est un test de l'ajustement de la sensibilité des deux récepteurs.

Les autres enregistrements permettent de contrôler la réponse du stabilisateur à divers régimes de fluctuations: variations brusques et de grande amplitude (Fig. 1b), dérive lente (Fig. 1c).

Dans tous les cas, le bruit est éliminé. Des variations de grande amplitude $( \pm 15 \%)$ mais relativement lentes sont bien corrigées : la fluctuation résiduelle est inférieure à $\pm 1 \%$. Par contre, les variations brusques sont partiellement transmises (fluctuations résiduelles de l'ordre de $2 \%$, par suite du temps de réponse relativement élevé de la cellule photorésistante $(0,5 \mathrm{~s}$ environ).

3.3 TransparenCe DU DisPositif. - Les pertes de luminosité sont dues à deux causes :

- En l'absence de champ appliqué, la transparence du dispositif est de $85 \%$ environ : ceci provient des pertes par réflexion sur les galets de la cloche contenant le KDP et sur le KDP lui-même ; on pourrait les diminuer par l'emploi de couches anti-réfléchissantes sur les galets.

- Le principe de la régulation consistant à « mettre en réserve" une partie du flux pour corriger les variations du laser entraîne une diminution du flux moyen transmis d'autant plus élevée que les fluctuations sont plus importantes. Dans les expériences décrites, la perte moyenne est de l'ordre de $15 \%$.

Dans ces conditions, la transparence du dispositif est de $70 \%$.

4. Conclusion. - Le dispositif décrit fournit une solution simple au problème de la stabilisation du flux transporté par un faisceau laser. Une perte de luminosité de l'ordre de $30 \%$ permet de corriger des variations de $\pm 15 \%$ de la puissance émise. A la limite, au prix d'une perte de $50 \%$, des variations de $\pm 50 \%$ peuvent être éliminées.

Remerciements. - Nous sommes très reconnaissants à M. P. Berge du Service de Physique du Solide et de Résonance Magnétique, CEN, Saclay, qui nous a proposé la réalisation de ce dispositif et prêté l'échantillon de KDP.
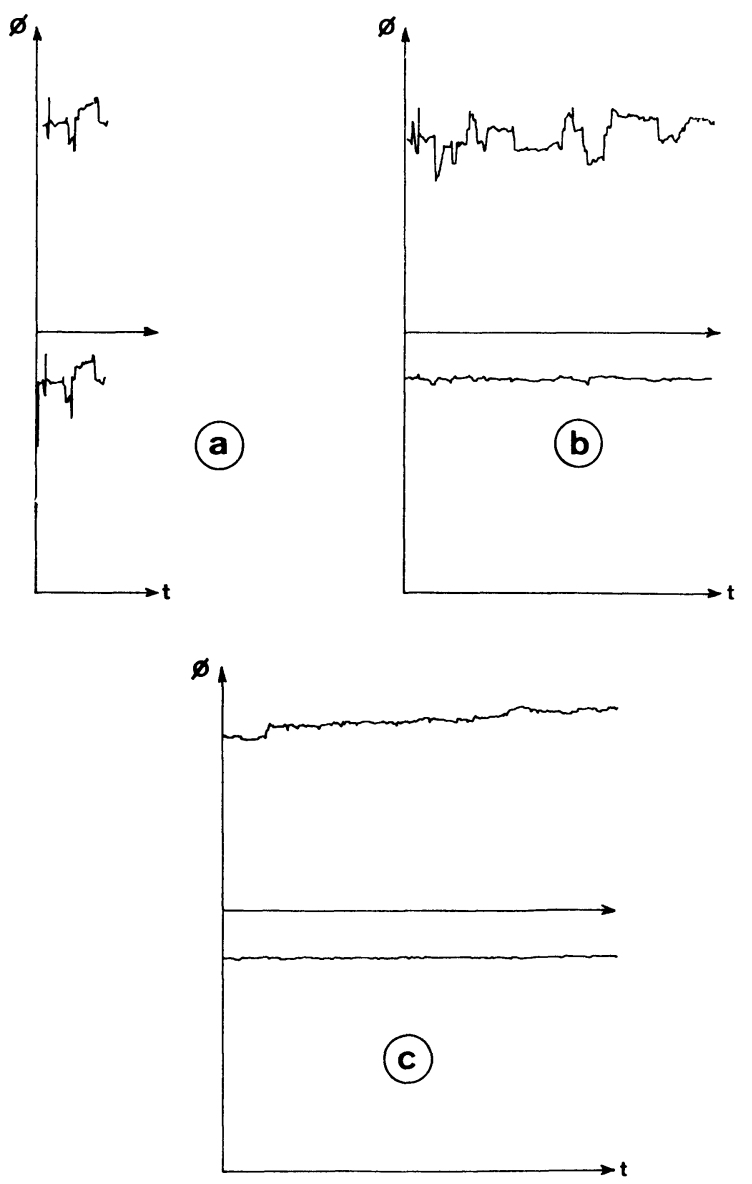

Fig. 1. - a) Sans correction, b) c) Fonctionnement du régulateur en présence de différents régimes de fluctuations.

\section{Bibliographie}

[1] Le Mezec, Henaff, Nguyen Ngoc Chan, L'onde électrique 46 (1966) 517. 